\title{
A Comparative Analysis of two Controllers for Trajectory Tracking Control: Application to a Biological Process
}

\author{
Abyad Mohamed ${ }^{\Uparrow}$, Karama Asma, Khallouq Abdelmounaim \\ LAEPT-URAC 28, Faculty of Science Semlalia, Cadi Ayyad University, Marrakech, Morocco
}

A R T I C L E I N F O

Article history:

Received: 01 June, 2018

Accepted: 04 August, 2018

Online: 20 August, 2018

\section{Keywords:}

Bioprocess

Constraints

Fuzzy observer

LMIs

Takagi-Sugeno Model

Trajectory tracking

\begin{abstract}
A B S T R A C T
The aim of the present work is to guarantee the trajectory tracking of a nonlinear biological process and compare two control approaches. The main objective of this work is to elaborate a fuzzy model and build a fuzzy controllers for a biological process by using the fuzzy Takagi-Sugeno. Two controllers are synthesized, the parallel distributed compensation control and optimal fuzzy linear quadratic integral control. In both cases, the physical constraints on the manipulated inputs are respected. In addition, the case with and without the observer is presented, where a fuzzy observer based control is used with unmeasurable premise variables. Finally, the performances and the effectiveness of both the modeling and the control are demonstrated via simulations.
\end{abstract}

\section{Introduction}

Nowadays, the biological processes become one of the important industrial processes thanks to their advantages, such as the treatment of organic substrates, protein production and the production of ethanol gas etc. However, their modeling and control form a real challenge problem for both control engineers and theorists, where this kind of systems are characterized by strong variations of system parameters and unknown kinetics owing to the time-varying characteristics and multiple interactions generated by the living microorganisms [1, 2]. Therefore, we obtain a highly nonlinear system. The motivation of this work is to linearize the model and benefit from linear theory control and to try to develop a nonlinear control, which is very difficult in this case. Also to use the Takagi-Sugeno (T-S) model, furthermore, the proposed controllers can be applied to the real process. It only needs to identify a T-S model from experimental data. T-S approach has been recognized as an effective tool for handling the previous difficulty.

There are different techniques for controlling the bioprocess using Takagi-Sugeno models, such as optimal fuzzy linear quadratic regulators for discrete-time [3], a fuzzy integral controller to force the switching of a bioprocess between two different metabolic states is

${ }^{*}$ Corresponding Author: Abyad Mohamed, Faculty of Science Semlalia Marrakech , Morocco, abymedlmp@gmail.com treated in [4], an internal model control design strategy is developed for a particular Continuous Stirred-Tank Reactor (CSTR) [5]. A PID and fuzzy controller are proposed in [6] to stabilize the CSTR around the equilibrium point, where the authors consider only one input, which is not the case in practice. Also, the case of uncertain Takagi-Sugeno system is treated in [7], where an observer with unmeasurable premise variables and unknown input is considered for a wastewater treatment plant. In addition, the predictive control based on fuzzy observer is studied for a sludge depollution bioprocess in [8, 9], in this framework one can cite $[10,11,12]$. Furthermore, the modeling and the control of bioprocess based on neural network approach is treated in [13, 14]. In the same spirit, a nonlinear model autoregressive with exogenous input model predictive control is developed in [15] to control the fermentation process. Also, an integral backstepping control law is developed in [16] for controlling the dissolved oxygen level for bacteria fermentation.

The problem treated in this paper is how to model and control the biomass growth process, ensuring the trajectory tracking while taking into account the following constraints:

- The mathematical model is nonlinear and not affine in control.

- The variables control present the physical constraints, 
which make the computation of the control gains difficult.

- The full system states are not measurable.

The present paper has two goals, the first is to build a fuzzy model of biological process based on TakagiSugeno tool, especially the nonlinearity sector methods. The second is to ensure the tracking trajectory of the desired outputs using two approaches: the Parallel Distributed Compensation (PDC) [17 and the Linear Quadratic Integral (LQI) control. Where the strong physical constraints on the inputs [18] are taking into account. In addition, the proposed controllers are compared. The stability conditions are formed in the Linear Matrix Inequalities (LMIs) terms.

This paper is organized as follows: Section 2 presents the description of the Takagi-Sugeno modeling. Section 3 describes the parallel distributed compensation control. Then, one can address the fuzzy output tracking control problem in section 4 and we show that it can be solved by using two methods: the PDC technique and the optimal linear quadratic control. Section 5 describes the controller design based on fuzzy observer with unmeasurable premise variables. Section 6 introduces the proposed biological process. Finally, the simulation and the discussion of the obtained results are given to compare the proposed controllers.

\section{Takagi-Sugeno Fuzzy Model}

In order to extend the existing approaches of control and observation for linear to nonlinear system, Takagi and Sugeno have proposed a fuzzy dynamic model to represent this kind of system. The T-S fuzzy model is a set of linear models connecting via membership functions. To build the T-S fuzzy model, three methods exist in the literature [17]: The black box identification, the linearization method and the nonlinearity sector methods. The third method gives an exact T-S representation of nonlinear system without information loss.

Consider the following nonlinear system:

$$
\begin{aligned}
& \dot{x}(t)=f(x(t))+g(x(t)) u(t) \\
& y(t)=C x(t)
\end{aligned}
$$

where $x \in \mathbb{R}^{n}$ is the state, $u \in \mathbb{R}^{m}$ is the input vector, $y \in \mathbb{R}^{q}$ represents the output measurement vectors and $C \in \mathbb{R}^{q * n}$ is the output matrix. In addition, $f($.$) and g($. represent the nonlinear functions.

The T-S fuzzy model uses a set of fuzzy if-then rules, which represent local linear input-output relations of a nonlinear system. The $i^{\text {th }}$ rule of the T-S model given as follows:

Rule i:

if $z_{1}(t)$ is $F_{1}^{i}\left(z_{1}(t)\right)$ and $z_{2}(t)$ is $F_{2}^{i}\left(z_{2}(t)\right) \ldots$ and $z_{p}(t)$ is $F_{p}^{i}\left(z_{p}(t)\right)$

$$
\text { Then }\left\{\begin{array}{l}
\dot{x}(t)=A_{i} x(t)+B_{i} u(t) \\
y(t)=C_{i} x(t)
\end{array}\right.
$$

Where $F_{p}^{i}$ are the membership functions of fuzzy sets, $i \in\{1,2, \ldots r\}, r$ is the number of rules, $A_{i} \in \mathbb{R}^{n * n}$, $B_{i} \in \mathbb{R}^{n * m}, C_{i} \in \mathbb{R}^{q * n}$ and $z_{1}(t), \ldots, z_{p}(t)$ are the premise variables which can be dependent of the input, the output or the state. The global T-S fuzzy model is given in the following form:

$$
\begin{aligned}
& \dot{x}(t)=\sum_{i=1}^{r} h_{i}(z(t))\left(A_{i} x(t)+B_{i} u(t)\right) \\
& y(t)=C x(t)
\end{aligned}
$$

where

$$
h_{i}(z(t))=\frac{\prod_{j=1}^{p} F_{j}^{i}\left(z_{j}(t)\right)}{\sum_{i=1}^{r} \prod_{j=1}^{p} F_{j}^{i}\left(z_{j}(t)\right)}
$$

The activation functions $h_{i}(z(t))$ indicates the activation degree of the $i^{t h}$ associated local model, this functions verifies all time the convex sum propriety:

$$
\left\{\begin{array}{l}
0 \leq h_{i}(z(t)) \leq 1 \\
\sum_{i=1}^{r} h_{i}(z(t))=1, \forall i \in\{1,2, \ldots, r\} .
\end{array}\right.
$$

\section{PDC control approach}

\subsection{Fuzzy regulator design via PDC}

To stabilize the system presented by their T-S fuzzy model, the PDC controller is usually used to design a fuzzy controller. The main idea is to design a local controller for each sub-model based on local control rule, which shares with the fuzzy model the same fuzzy sets. The overall fuzzy controller is represented by:

$$
u(t)=-\sum_{i=1}^{r} h_{i}(z) K_{i} x(t)
$$

Where the $K_{i}$ represent the local feedback gains. by using (6) in (3) the system in closed-loop becomes:

$$
\begin{aligned}
\dot{x}(t) & =\sum_{i=1}^{r} \sum_{j=1}^{r} h_{i}(z) h_{j}(z)\left(A_{i}-B_{i} K_{j}\right) x(t) \\
& =\sum_{i=1}^{r} h_{i}^{2}(z) G_{i i} x(t)+2 \sum_{i<j}^{r} h_{i}(z) h_{j}(z)\left(\frac{G_{i j}+G_{j i}}{2}\right) x(t)
\end{aligned}
$$

with $G_{i j}=A_{i}-B_{i} K_{j}$, the stability conditions of (7) are given by the following theorem [17].

Theorem 1 The continuous fuzzy system (7) is asymptotically stable, if there exist a common positive matrix $P \in R^{n \times n}$ and a common positive semi definite matrix $Q \in R^{n \times n}$ and for a number of active rules $s$, where $1<s \leq r$ such that:

$$
\begin{array}{r}
G_{i i}^{T} P+P G_{i i}+(s-1) Q<0 \\
\left(\frac{G_{i j}+G_{j i}}{2}\right)^{T} P+P\left(\frac{G_{i j}+G_{j i}}{2}\right)-Q \leq 0, i<j
\end{array}
$$

where $s>1$. 
In order to transform the preview conditions into LMIs form, one can consider the following variables: $X=P^{-1}$ , $K_{i}=M_{i} X^{-1}, Q=P Y P$, where $X>0, Y \geq 0$ and $M_{i}(i=1, \ldots, r)$, then the stabilization conditions become:

$$
\begin{aligned}
& A_{i} X-B_{i} M_{i}+X A_{i}^{T}-M_{i}^{T} B_{i}^{T}+(s-1) Y<0 \\
& A_{i} X-B_{i} M_{j}+X A_{i}^{T}-M_{j}^{T} B_{i}^{T}+A_{j} X-B_{j} M_{i} \\
& +X A_{j}^{T}-M_{i}^{T} B_{j}^{T}-2 Y \leq 0, \quad i<j
\end{aligned}
$$

\section{Trajectory tracking control}

The trajectory tracking control of nonlinear systems is the subject this section. In the tracking loop, we consider the integral of the tracking error $e_{I}=\int\left(y_{r}-y\right) d t=$ $\int\left(y_{r}-C x\right) d t$ [19], with $y_{r}$ is the desired output. If we consider the following augmented state:

$$
X_{a}=\left[\begin{array}{c}
x \\
e_{I}
\end{array}\right]
$$

Then, the following augmented system is obtained:

$$
\left\{\begin{array}{l}
\dot{X}_{a}(t)=\sum_{i=1}^{r} h_{i}(z(t))\left(\bar{A}_{i} X_{a}(t)+\bar{B}_{i} u(t)+\bar{D} Y_{r}\right) \\
y(t)=\bar{C} X_{a}(t)
\end{array}\right.
$$

where: $\bar{A}_{i}=\left[\begin{array}{cc}A_{i} & 0 \\ -C & 0\end{array}\right], \bar{B}_{i}=\left[\begin{array}{c}B_{i} \\ 0\end{array}\right], \bar{C}=\left[\begin{array}{ll}C & 0\end{array}\right], \bar{D}=\left[\begin{array}{l}0 \\ \text { I }\end{array}\right]$, $Y_{r}=\left[\begin{array}{l}y_{r 1} \\ y_{r 2}\end{array}\right]$

with $Y_{r}$, denote the desired reference trajectory.

\subsection{PDC control}

To achieve the output tracking, the state feedback PDC control based on the previous LMIs can be used. The fuzzy controller $u(t)$ has the same form of $(6)$, where $x$ is replaced by the augmented state $X_{a}$ :

$$
u(t)=-\sum_{i=1}^{r} h_{i}(z) K_{i} X_{a}(t)=-\sum_{i=1}^{r} h_{i}(z)\left(K_{i x} x+K_{i I} e_{I}\right)
$$

The feedback gains of the controller $K_{i}=\left[\begin{array}{ll}K_{i x} & K_{i I}\end{array}\right]$ are obtained by solving the LMIs 10 .

\subsection{LQI control}

To design the LQI control, the following quadratic cost criterion must be minimized by the control law $u(t)$ :

$$
J=\int_{0}^{\infty}\left(X_{a}^{T}(t) Q X_{a}(t)+u^{T}(t) R u(t)\right) d t
$$

for this reason, the following candidate quadratic Lyapunov function is considered:

$$
V\left(X_{a}\right)=X_{a}^{T} P X_{a}
$$

The augmented system (7) is stable if :

$$
X_{a}^{T} Q X_{a}+u^{T} R u+\dot{V}\left(X_{a}\right)<0
$$

$$
\begin{gathered}
\left(\overline{A_{i}}+\overline{B_{i}} K_{i}\right)^{T} P_{i}+P_{i}\left(\overline{A_{i}}+\overline{B_{i}} K_{i}\right)+Q+K_{i}^{T} R K_{i}<0 \\
\left(\overline{A_{i}} X_{i}+\overline{B_{i}} Y_{i}\right)^{T}+\left(\overline{A_{i}} X_{i}+\overline{B_{i}} Y_{i}\right)+X_{i} Q X_{i}+Y_{i}^{T} R Y_{i}<0 \\
\left(\overline{A_{i}} X_{i}+\bar{B}_{i} Y_{i}\right)^{T}+\left(\overline{A_{i}} X_{i}+\bar{B}_{i} Y_{i}\right)+Y_{i}^{T} R Y_{i}-X_{i}(-Q) X_{i}<0
\end{gathered}
$$

by using the Schur complement procedure, the following stability conditions are obtained:

$$
\left[\begin{array}{ccc}
\left(\bar{A}_{i} X_{i}+\bar{B}_{i} Y_{i}\right)^{T}+\left(\bar{A}_{i} X_{i}+\bar{B}_{i} Y_{i}\right) & X_{i} & Y_{i}^{T} \\
* & -\tilde{Q} & 0 \\
* & * & -\tilde{R}
\end{array}\right]<0
$$

where: $Q^{-1}=\tilde{Q}, R^{-1}=\tilde{R}, X_{i}=P_{i}^{-1}, K_{i}=Y_{i} X_{i}^{-1}$, $i=1, \ldots, r$

This LMIs will be calculated for each sub-model, here is about the conventional linear quadratic integral control using the fuzzy model. The control law is not based on fuzzy rules.

The control law will be respect the physical constraints on the control input, where this problem is studied in many practical cases [20, 21, 22, 23]. To ensure the stabilization under constraints on the inputs, the conditions given in the following theorem [17] must be verified.

Theorem 2 For a known initial condition $X_{a}(0)$, the constraint $\|u(t)\|_{2} \leq \eta$ is enforced at all times $t \geq 0$ if the LMIs:

$$
\begin{aligned}
{\left[\begin{array}{cc}
1 & X_{a}(0)^{T} \\
X_{a}(0) & X
\end{array}\right] } & \geq 0 \\
{\left[\begin{array}{cc}
X & M_{i}^{T} \\
M_{i} & \eta^{2} I
\end{array}\right] } & \geq 0
\end{aligned}
$$

hold

\subsection{Observer design}

In bioprocess control problems, the state variables are not usually available. By introducing the observer, one can reconstruct partially or all the state variables. This section presents the fuzzy observer design with unmeasurable premise variables $z(t)\left(h_{i}(z) \neq h_{i}(\hat{z})\right)$.

Based on the structure of the fuzzy model (3), the fuzzy observer is given as follows:

$$
\left\{\begin{array}{l}
\dot{\hat{x}}(t)=\sum_{i=1}^{r} h_{i}(\hat{z})\left(A_{i} \hat{x}(t)+B_{i} u(t)+L_{i}(y(t)-\hat{y}(t))\right. \\
\hat{y}(t)=\sum_{i=1}^{r} h_{i}(\hat{z}) C_{i} \hat{x}(t)
\end{array}\right.
$$

where $\hat{x}$ denotes the estimated state and $L_{i}$ the gains of the observer.

In order to compute this gains the following theorem [24] gives the necessary conditions for ensuring the convergence of the state estimation error to zero.

Theorem 3 If there exist symmetric and definite positive matrices $P \in \mathbb{R}^{n \times n}, Q \in \mathbb{R}^{n \times n}$, matrices $Y_{i} \in \mathbb{R}^{n \times q}$ and $a$ scalar $\alpha>0$ such that:

$$
\begin{array}{r}
A_{i}^{T} P+P A_{i}-C^{T} Y_{i}^{T}-Y_{i}^{T} C \leq-Q \\
{\left[\begin{array}{cc}
Q-\alpha^{2} \mathbb{I} & P \\
P & \mathbb{I}
\end{array}\right]>0}
\end{array}
$$


then, the estimation error between the T-S fuzzy model 3 and the fuzzy observer 22, is converges asymptotically to zero.

where: $L_{i}=P^{-1} Y_{i}$.

\section{Proof 1 See Appendix.}

\section{Process description}

The proposed biological process in this paper is a biomass growth process, which consists to grow the population of microorganisms (biomass) by the consumption of a substrate (glucose), according to the following reaction scheme:

$$
k_{1} S \stackrel{\mu(.)}{\longmapsto} X
$$

The dynamic model of this process is established from the mass-balance [25], which describes the evolution of substrate and biomass concentrations in a continuous bioreactor. This model can be represented by a high nonlinear system as follows:

$$
\left\{\begin{array}{l}
\frac{d X}{d t}=\mu(.) X-D X \\
\frac{d S}{d t}=-k_{1} \mu(.) X+D\left(S_{i n}-S\right)
\end{array}\right.
$$

The state variables are the biomass $X$ and substrate $S$ concentrations, $k_{1}$ denotes the pseudo stoichiometric coefficient and $\mu($.) represent the specific growth rate, the "Monod law" characterizes $\mu($.$) is:$

$$
\mu(S)=\mu_{\max } \frac{S}{K_{S}+S}
$$

where $\mu_{\text {max }}$ is the maximum specific growth rate; $K_{s}$ is the Monod or saturation constant. The input variables are the dilution rate $D(t)$ and the influent substrate concentration $S_{i n}$. The parameters of the proposed model are given in the Table 1 .

\begin{tabular}{|c|c|c|}
\hline Parameters & Value & Unit \\
\hline$\mu_{\max }$ & 0.38 & $h^{-1}$ \\
\hline$K_{s}$ & 5 & $g / l$ \\
\hline$k_{1}$ & $1 / 0.07$ & \\
\hline$S_{i n}^{\max }$ & 140 & $g / l$ \\
\hline
\end{tabular}

Table 1: Simulation parameters

\subsection{Takagi-Sugeno model design}

The model 26 must be transformed into affine in control model like in (1), where the bioprocess models are known belong to the class of affine nonlinear models, this can be easily shown by assuming that:

$$
\begin{aligned}
D & =D_{1}+D_{2} \\
S_{i n} & =\frac{D_{2}}{D_{1}+D_{2}} S_{i n}^{\text {max }}
\end{aligned}
$$

where $D_{1}(t)$ and $D_{2}(t)$ are respectively the water and the substrate dilution rate, then one can replace $D(t)$ and $S_{i n}(t)$ in 26 by their expressions 28, the following affine model is obtained:

$$
\left[\begin{array}{l}
\frac{d X}{d t} \\
\frac{d S}{d t}
\end{array}\right]=\left[\begin{array}{c}
\mu(S) X \\
-k_{1} \mu(S) X
\end{array}\right]+\left[\begin{array}{cc}
-X & -X \\
-S & S_{i n}^{\max }-S
\end{array}\right]\left[\begin{array}{c}
D_{1} \\
D_{2}
\end{array}\right]
$$

where:

$$
\begin{gathered}
f(x)=\left[\begin{array}{c}
\mu(S) X \\
-k_{1} \mu(S) X
\end{array}\right], g(x)=\left[\begin{array}{cc}
-X & -X \\
-S & S_{i n}^{\max }-S
\end{array}\right] \\
x(t)=\left[\begin{array}{l}
X \\
S
\end{array}\right] \text { and } u(t)=\left[\begin{array}{l}
D_{1} \\
D_{2}
\end{array}\right]
\end{gathered}
$$

To build the T-S model, the following nonlinearities are considered:

$$
\begin{aligned}
& z_{1}(x)=\mu(S) \\
& z_{2}(x)=X \\
& z_{3}(x)=S
\end{aligned}
$$

This leads:

$$
A(z)=\left[\begin{array}{cc}
z_{1} & 0 \\
-k_{1} z_{1} & 0
\end{array}\right] \text { and } B(z)=\left[\begin{array}{cc}
-z_{2} & -z_{2} \\
-z_{3} & -z_{3}+S_{\text {in }}^{\max }
\end{array}\right]
$$

where the number of nonlinarities $n=3$, the global model can be represented by $r=2^{n}=8$ sub-models. The local membership functions are defined by:

$$
\begin{aligned}
& F_{1}^{1}\left(z_{1}\right)=\frac{z_{1}-z_{1}^{\min }}{z_{1}^{\max }-z_{1}^{\min }}, \quad F_{1}^{2}\left(z_{1}\right)=\frac{z_{1}^{\max }-z_{1}}{z_{1}^{\max }-z_{1}^{\min }} \\
& F_{2}^{1}\left(z_{2}\right)=\frac{z_{2}-z_{2}^{\min }}{z_{2}^{\max }-z_{2}^{\min }}, \quad F_{2}^{2}\left(z_{2}\right)=\frac{z_{2}^{\max }-z_{2}}{z_{2}^{\max }-z_{2}^{\min }} \\
& F_{3}^{1}\left(z_{3}\right)=\frac{z_{3}-z_{3}^{\min }}{z_{3}^{\max }-z_{3}^{\min }}, \quad F_{3}^{2}\left(z_{3}\right)=\frac{z_{3}^{\max }-z_{3}}{z_{3}^{\max }-z_{3}^{\min }}
\end{aligned}
$$

Finally, the activation functions are:

$$
\begin{array}{ll}
h_{1}(z)=F_{1}^{1}\left(z_{1}\right) F_{2}^{1}\left(z_{2}\right) F_{3}^{1}\left(z_{3}\right), & h_{2}(z)=F_{1}^{1}\left(z_{1}\right) F_{2}^{1}\left(z_{2}\right) F_{3}^{2}\left(z_{3}\right) \\
h_{3}(z)=F_{1}^{1}\left(z_{1}\right) F_{2}^{2}\left(z_{2}\right) F_{3}^{1}\left(z_{3}\right), & h_{4}(z)=F_{1}^{1}\left(z_{1}\right) F_{2}^{2}\left(z_{2}\right) F_{3}^{2}\left(z_{3}\right) \\
h_{5}(z)=F_{1}^{2}\left(z_{1}\right) F_{2}^{1}\left(z_{2}\right) F_{3}^{1}\left(z_{3}\right), & h_{6}(z)=F_{1}^{1}\left(z_{1}\right) F_{2}^{1}\left(z_{2}\right) F_{3}^{2}\left(z_{3}\right) \\
h_{7}(z)=F_{1}^{2}\left(z_{1}\right) F_{2}^{2}\left(z_{2}\right) F_{3}^{1}\left(z_{3}\right), & h_{8}(z)=F_{1}^{2}\left(z_{1}\right) F_{2}^{2}\left(z_{2}\right) F_{3}^{2}\left(z_{3}\right)
\end{array}
$$

For the simulation, the parameters given in Table 1 are considered and leads to the following min and max of premise variables:

$$
\begin{array}{ll}
0.018 & \leq \mu(S) \leq 0.35 \\
3.8 & \leq X \leq 20 \\
0.6 & \leq S \leq 140
\end{array}
$$

the computed matrix $A_{i}$ and $B_{i}$ of each sub-model are given as follows:

$$
\begin{aligned}
& A_{1}=A_{3}=A_{5}=A_{7}=\left[\begin{array}{cc}
0.3507 & 0 \\
-5.0106 & 0
\end{array}\right] \\
& A_{2}=A_{4}=A_{6}=A_{8}=\left[\begin{array}{cc}
0.0179 & 0 \\
-0.2551 & 0
\end{array}\right] \\
& B_{1}=B_{2}=\left[\begin{array}{cc}
-20 & -20 \\
-140 & 0
\end{array}\right], B_{3}=B_{4}=\left[\begin{array}{cc}
-3.8 & -3.8 \\
-140 & 0
\end{array}\right] \\
& B_{5}=B_{6}=\left[\begin{array}{cc}
-20 & -20 \\
-0.6 & 139.4
\end{array}\right], B_{7}=B_{8}=\left[\begin{array}{cc}
-3.8 & -3.8 \\
-0.60 & 139.4
\end{array}\right]
\end{aligned}
$$




\section{Simulation and results}

In the first case, all the states variables (substrate and biomass concentrations) are supposed measurable (i.e. $\left.C=\left[\begin{array}{ll}1 & 0 \\ 0 & 1\end{array}\right]\right)$.

The desired trajectory $\left(X_{r}\right.$ and $\left.S_{r}\right)$, which represent respectively of biomass and substrate concentrations are computed by using the following reference model:

$$
\begin{aligned}
& \dot{X}_{r}=-0.97 X_{r}+0.97 r e f_{X} \\
& \dot{S}_{r}=-0.65 S_{r}+0.65 r e f_{S}
\end{aligned}
$$

where $r e f_{X}$ and $r e f_{S}$ are the setpoints.

\subsection{Tracking control based on state feed- back}

\subsubsection{PDC control}

The studied bioprocess presents the physical constraints on the control as shown in the Table 2

\begin{tabular}{|c|c|}
\hline Variables & Constraints \\
\hline Dilution rate $h^{-1}$ & $0.01 \leqslant D \leqslant 0.38$ \\
\hline Influent substrate $g / l$ & $60 \leqslant S_{i n} \leqslant 140$ \\
\hline
\end{tabular}

Table 2: The control constraints

For a number of active sub-model $s=5$ and $\eta=$ 1.55 , the LMIs (10), 20) and 211 are solved by using the solver SeDuMi in MATLAB toolbox YALMIP, gives the following gains:

$$
\begin{aligned}
K_{1} & =\left[\begin{array}{cccc}
0.0384 & -0.0044 & -0.0007 & 0.0083 \\
-0.2821 & 0.0064 & 0.0564 & -0.0088
\end{array}\right] \\
K_{2} & =\left[\begin{array}{cccc}
0.0052 & -0.0044 & -0.0007 & 0.0083 \\
-0.2472 & 0.0064 & 0.0563 & -0.0088
\end{array}\right] \\
K_{3} & =\left[\begin{array}{cccc}
0.03470 & -0.0044 & 0.0002 & 0.0083 \\
-0.2963 & 0.0064 & 0.05930 & -0.0088
\end{array}\right] \\
K_{4} & =\left[\begin{array}{cccc}
0.0011 & -0.0044 & 0.0002 & 0.0083 \\
-0.2605 & 0.0064 & 0.05910 & -0.0088
\end{array}\right] \\
K_{5} & =\left[\begin{array}{cccc}
-0.2149 & -0.0010 & 0.0572 & 0.0074 \\
-0.0287 & 0.0044 & -0.0014 & -0.0083
\end{array}\right] \\
K_{6} & =\left[\begin{array}{cccc}
-0.2480 & -0.0010 & 0.0571 & 0.0074 \\
0.0060 & 0.0044 & -0.0015 & -0.0083
\end{array}\right] \\
K_{7} & =\left[\begin{array}{cccc}
-0.22450 & -0.0009 & 0.0592 & 0.0074 \\
-0.0371 & 0.0044 & 0.0003 & -0.0083
\end{array}\right] \\
K_{8} & =\left[\begin{array}{cccc}
-0.25660 & -0.0009 & 0.0590 & 0.0074 \\
-0.0028 & 0.0044 & 0.0003 & -0.0083
\end{array}\right] \\
P & =\left[\begin{array}{cccc}
0.0059 & -0.0000 & -0.0011 & 0.0000 \\
-0.0000 & 0.0008 & 0.0000 & -0.0002 \\
-0.0011 & 0.0000 & 0.0008 & -0.0000 \\
0.0000 & -0.0002 & -0.0000 & 0.0008
\end{array}\right] \\
Q & =\left[\begin{array}{cccc}
7.5254 & 0.0002 & 2.9997 & -0.0011 \\
0.0002 & 35.5015 & 0.0002 & 0.0002 \\
2.9997 & 0.0002 & 23.9724 & 0.0013 \\
-0.0011 & 0.0002 & 0.0013 & 26.1931
\end{array}\right]
\end{aligned}
$$

The initial conditions are $x_{0}=\left(\begin{array}{ll}6.6 & 5.50\end{array}\right)^{T}$ and the obtained results are shown in Figures 1 and 2 , the trajectory tracking is achieved, where the biomass and the substrate concentrations follow the desired outputs. In addition, the constraints on the inputs control $D(t)$ and $S_{i n}(t)$ are respected.
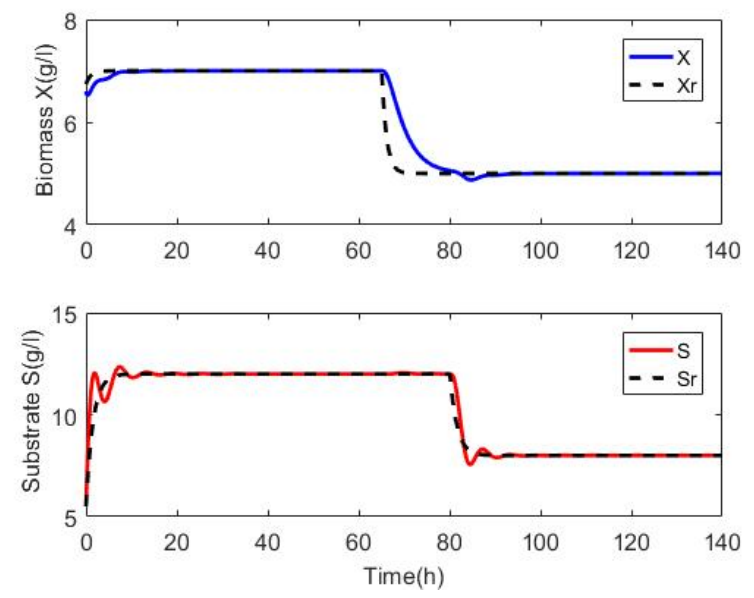

Figure 1: Evolution of the system outputs
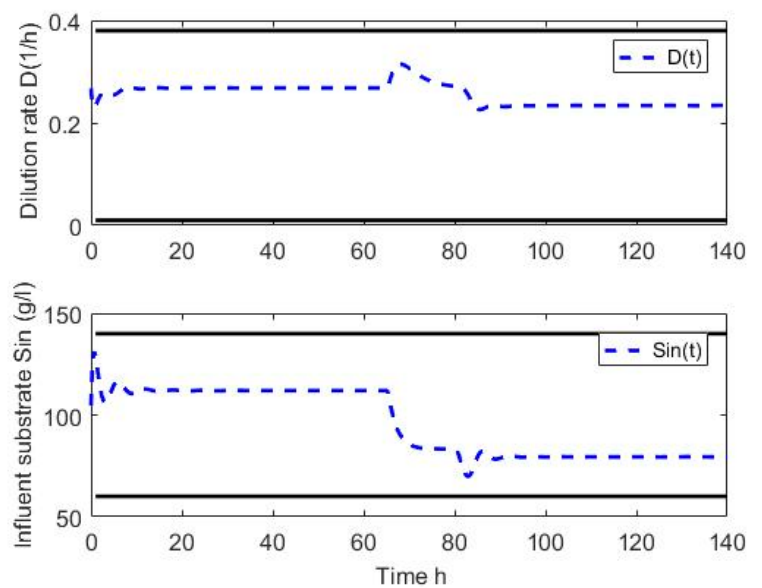

Figure 2: Control inputs $D(t)$ and $S_{i n}(t)$

\subsubsection{LQI control}

Solving the LMIs established in (19, 20) and 21) the obtained weighting matrices are:

$$
\begin{aligned}
R & =\left[\begin{array}{ll}
0.328 & 0.000 \\
0.000 & 0.328
\end{array}\right] \cdot 10^{-3} \\
Q & =\left[\begin{array}{cccc}
0.2529 & 0.0025 & -0.0264 & -0.0007 \\
0.0025 & 0.2252 & -0.0004 & -0.0422 \\
-0.0264 & -0.0004 & 0.1345 & 0.0015 \\
-0.0007 & -0.0422 & 0.0015 & 0.1966
\end{array}\right] \cdot 10^{-3}
\end{aligned}
$$

The controller gains are:

$$
K_{1}=\left[\begin{array}{cccc}
0.0202 & -0.0081 & 0.0044 & 0.0101 \\
-0.0736 & 0.0128 & 0.0098 & -0.0056
\end{array}\right]
$$




$$
\begin{aligned}
K_{2} & =\left[\begin{array}{cccc}
0.0010 & -0.0095 & 0.0010 & 0.0105 \\
-0.0368 & 0.0052 & 0.0195 & -0.0030
\end{array}\right] \\
K_{3} & =\left[\begin{array}{cccc}
0.0317 & -0.0095 & 0.0008 & 0.0106 \\
-0.2002 & 0.0039 & 0.0165 & -0.0004
\end{array}\right] \\
K_{4} & =\left[\begin{array}{cccc}
0.0016 & -0.0096 & 0.0002 & 0.0106 \\
-0.1086 & 0.0023 & 0.0271 & -0.0005
\end{array}\right] \\
K_{5} & =\left[\begin{array}{llll}
-0.0247 & -0.0003 & 0.0211 & 0.004 \\
-0.031 & 0.0095 & -0.0006 & -0.009
\end{array}\right] \\
K_{6} & =\left[\begin{array}{llll}
-0.0345 & -0.0050 & 0.0200 & 0.0033 \\
-0.0024 & 0.0096 & 0.0009 & -0.0105
\end{array}\right] \\
K_{7} & =\left[\begin{array}{cccc}
-0.1471 & -0.0047 & 0.0211 & 0.0035 \\
-0.0369 & 0.0095 & 0.0003 & -0.0105
\end{array}\right] \\
K_{8} & =\left[\begin{array}{llll}
-0.1064 & -0.0026 & 0.0274 & 0.0009 \\
-0.0023 & 0.0096 & 0.0003 & -0.0107
\end{array}\right]
\end{aligned}
$$

The Figures 3 and 4 show the simulation results using LQI control, where the controlled outputs variables achieve the desired outputs, also the constraints on control are respected.
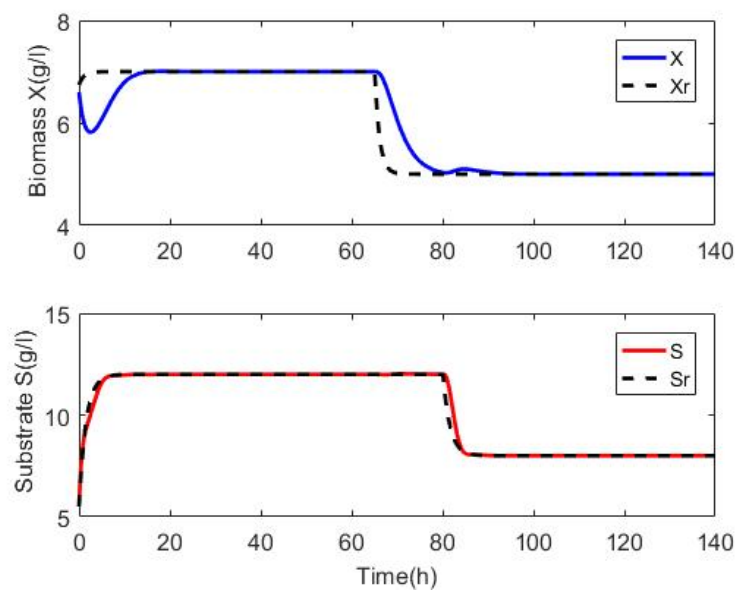

Figure 3: Evolution of the system outputs
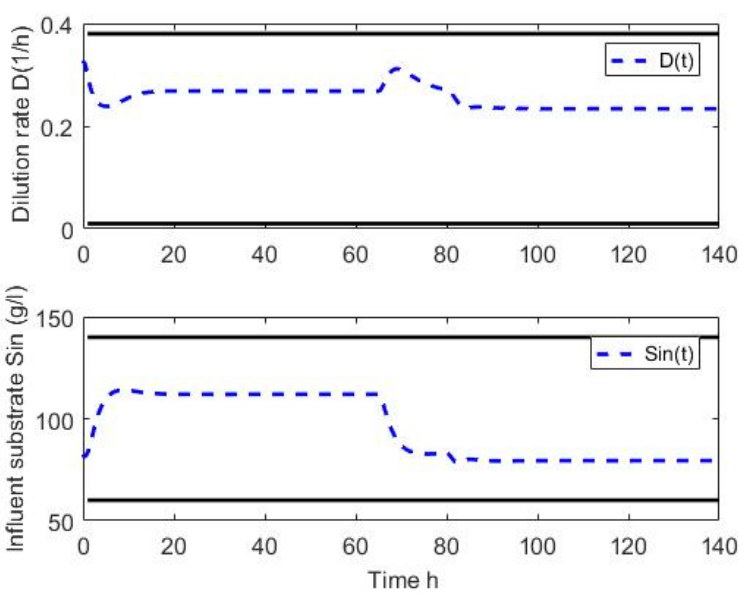

Figure 4: Control inputs $D(t)$ and $S_{i n}(t)$
The results obtained are comparable or even better than those obtained using the PDC controller.

\subsection{Tracking control based on recon- structed state feedback}

In the practical case, only the substrate concentration is measured and the biomass is estimated, then the output matrix in 11 becomes $C=\left[\begin{array}{ll}0 & 1\end{array}\right]$. For the initial conditions of system $x_{0}=\left(\begin{array}{ll}4 & 8\end{array}\right)^{T}$, the initial conditions of observer $\hat{x}_{0}=\left(\begin{array}{ll}5 & 7\end{array}\right)^{T}$ and for a scalar $\alpha=0.5$, the following observer gains are obtained:

$$
\begin{gathered}
L_{1}=L_{3}=L_{5}=L_{7}=\left[\begin{array}{c}
5.2458 \\
-1.3202
\end{array}\right] \\
L_{2}=L_{4}=L_{6}=L_{8}=\left[\begin{array}{c}
0.3483 \\
-0.6440
\end{array}\right] \\
Q=\left[\begin{array}{ccc}
5.655 & 0.000 & -0.000 \\
0.000 & 4.4141 & 0.000 \\
-0.00 & 0.000 & 6.2045
\end{array}\right] \\
P=\left[\begin{array}{lll}
0.5985 & 0.0843 & 0.0000 \\
0.0843 & 0.6103 & 0.0000 \\
0.0000 & 0.0000 & 0.6103
\end{array}\right]
\end{gathered}
$$

\subsubsection{PDC control}

The resolution of the same LMIs in last case gives the following gains.

$$
\begin{aligned}
K_{1} & =\left[\begin{array}{ccc}
0.0231 & -0.0043 & 0.0067 \\
-0.1294 & 0.0058 & -0.0037
\end{array}\right] \\
K_{2} & =\left[\begin{array}{llc}
-0.0057 & -0.0044 & 0.0066 \\
-0.1082 & 0.0058 & -0.0033
\end{array}\right] \\
K_{3} & =\left[\begin{array}{ccc}
0.0304 & -0.0044 & 0.0064 \\
-0.1425 & 0.0058 & -0.0027
\end{array}\right] \\
K_{4} & =\left[\begin{array}{lll}
-0.0038 & -0.0045 & 0.0065 \\
-0.1187 & 0.0058 & -0.0031
\end{array}\right] \\
K_{5} & =\left[\begin{array}{lll}
-0.0814 & -0.0015 & 0.0094 \\
-0.0336 & 0.0044 & -0.0065
\end{array}\right] \\
K_{6} & =\left[\begin{array}{lll}
-0.1090 & -0.0015 & 0.0092 \\
-0.0016 & 0.0044 & -0.0064
\end{array}\right] \\
K_{7} & =\left[\begin{array}{lll}
-0.0857 & -0.0018 & 0.0096 \\
-0.0359 & 0.0048 & -0.0065
\end{array}\right] \\
K_{8} & =\left[\begin{array}{lll}
-0.1151 & -0.0015 & 0.0094 \\
-0.0036 & 0.0046 & -0.0064
\end{array}\right] \\
P & =\left[\begin{array}{ccc}
0.0114 & -0.0001 & -0.0003 \\
-0.0001 & 0.0010 & -0.0002 \\
-0.0003 & -0.0002 & 0.0008
\end{array}\right] \\
Q & =\left[\begin{array}{ccc}
0.0114 & -0.0001 & -0.0003 \\
-0.0001 & 0.0010 & -0.0002 \\
-0.0003 & -0.0002 & 0.0008
\end{array}\right]
\end{aligned}
$$

The Figure 5 shows a comparison between the controlled variable $S$, his estimated $\hat{S}$ and the desired output $S_{r}$. The obtained result show that $S$ follows correctly $S_{r}$ and the observer estimates the states of 
system $(\hat{S}=S)$ after 4.33 hours. The Figure 6 presents the control inputs $D(t)$ and $S_{i n}(t)$, which respect the constraints.

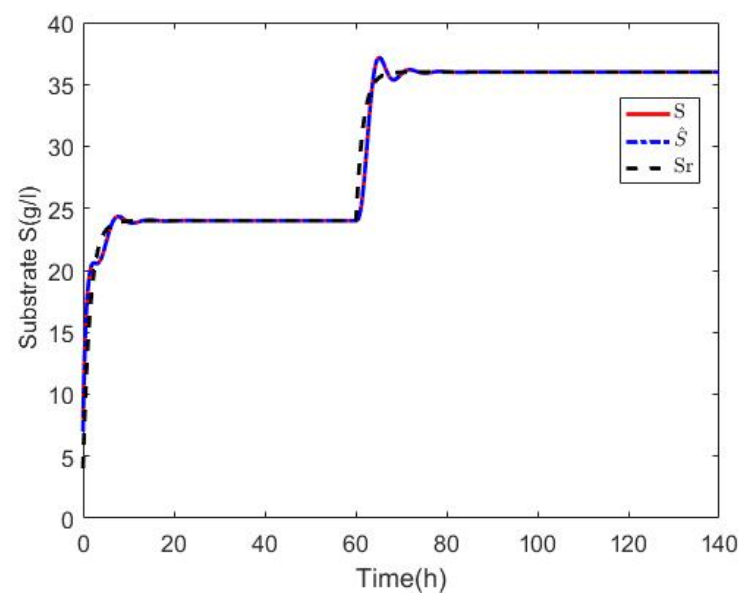

Figure 5: Evolution of the system outputs
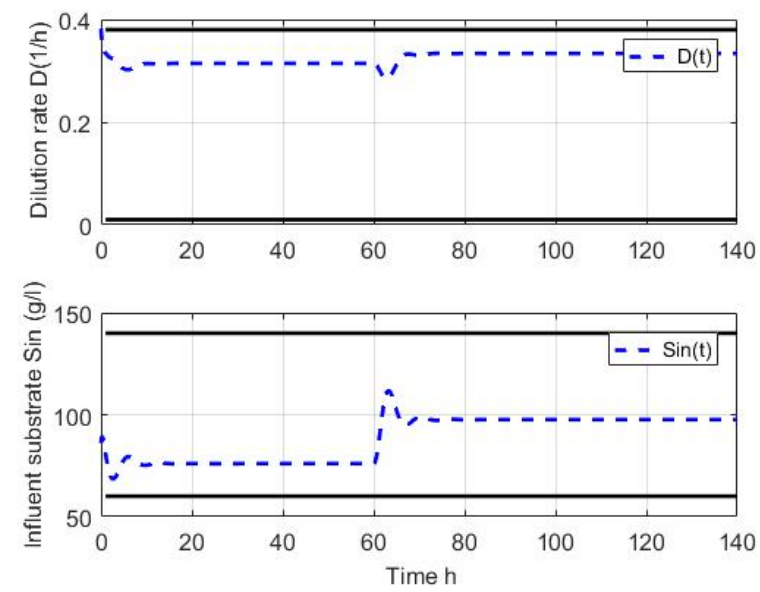

Figure 6: Control inputs $D(t)$ and $S_{i n}(t)$

\subsubsection{LQI control}

In this part, we illustrate the obtained results using LQI control. Solving the LMIs (20), 21), (19) we obtain the following controller gains and weighting matrices:

$$
\begin{aligned}
R & =\left[\begin{array}{ll}
0.2040 & 0.0002 \\
0.0002 & 0.2028
\end{array}\right] \cdot 10^{-3} \\
Q & =\left[\begin{array}{ccc}
0.1138 & 0.0012 & -0.0009 \\
0.0012 & 0.1360 & -0.0196 \\
-0.0009 & -0.0196 & 0.1072
\end{array}\right] .10^{-3} \\
K_{1} & =\left[\begin{array}{ccc}
0.0000 & -0.0043 & 0.0066 \\
-0.0566 & 0.0087 & -0.0035
\end{array}\right] \\
K_{2} & =\left[\begin{array}{ccc}
0.0015 & -0.0076 & 0.0067 \\
-0.0124 & 0.0015 & -0.0001
\end{array}\right] \\
K_{3} & =\left[\begin{array}{ccc}
0.0182 & -0.0072 & 0.0069 \\
-0.1308 & 0.0032 & -0.0003
\end{array}\right]
\end{aligned}
$$

$$
\begin{aligned}
K_{4} & =\left[\begin{array}{ccc}
0.0017 & -0.00760 & 0.0067 \\
-0.0222 & 0.0003 & 0.0002
\end{array}\right] \\
K_{5} & =\left[\begin{array}{llc}
-0.0130 & 0.0005 & 0.0032 \\
-0.0231 & 0.0071 & -0.00560
\end{array}\right] \\
K_{6} & =\left[\begin{array}{llc}
-0.0105 & -0.0017 & 0.0009 \\
-0.0016 & 0.0076 & -0.0067
\end{array}\right] \\
K_{7} & =\left[\begin{array}{llc}
-0.0624 & -0.0034 & 0.0027 \\
-0.0327 & 0.0073 & -0.0062
\end{array}\right] \\
K_{8} & =\left[\begin{array}{ccc}
-0.0204 & -0.0009 & 0.0004 \\
-0.0018 & 0.0076 & -0.0067
\end{array}\right]
\end{aligned}
$$

The Figures 7 and 8 present the same variables in the Figures 5 and 6 using the LQI control. where the obtained results indicate clearly that the desired performances can be achieved more better than the results obtained by PDC control, the Figure 9 shows the state estimation error of the state variables ( biomass and substrate concentrations), where this error tends to zero after 4.33 hours.

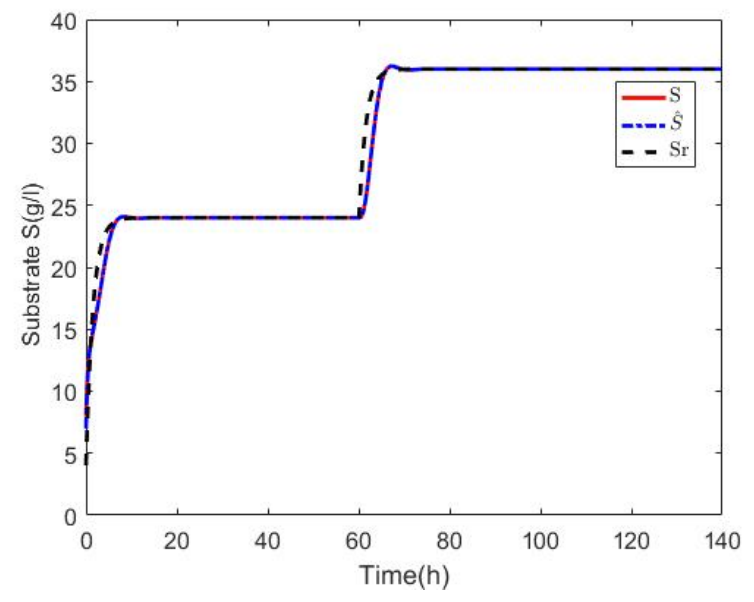

Figure 7: Evolution of the system outputs
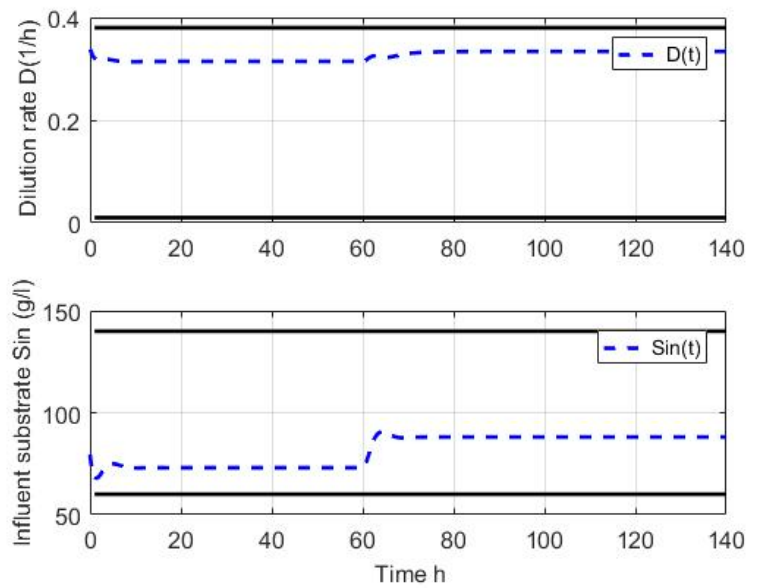

Figure 8: Control inputs $D(t)$ and $S_{i n}(t)$ 

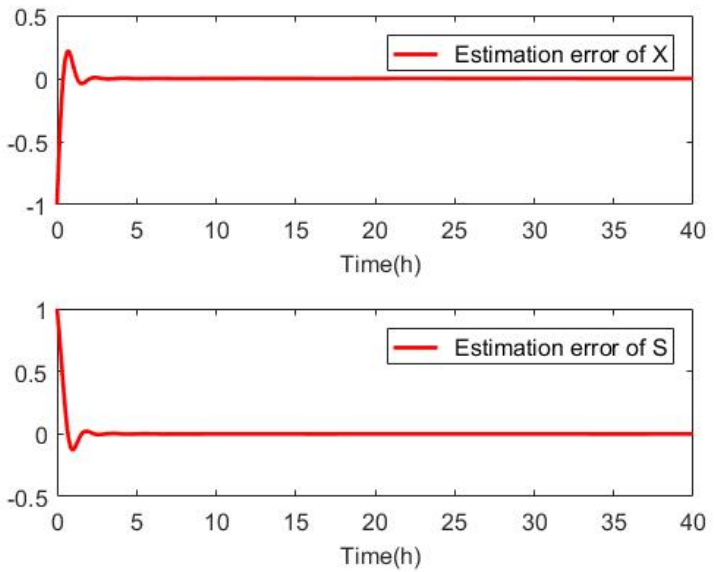

Figure 9: The sate estimation error

\subsection{Comparison of both controllers}

The outputs behaviors in the Figures 1, 3, 5, 7, show that the PDC controller presents some overshoot compared with LQI controller, where the PDC control requires the stabilization of all the different sub-models cross terms $\left(h_{i}(z) \neq h_{j}(z)\right)$ too, which increases the LMIs need to be solved. Moreover, in terms of speed, the comparison shows clearly that the LQI controller is fast than PDC one. In general, the comparison shows that the LQI controller presents the best performance.

\subsection{Comparison with other methods}

Another method of bioprocess control based on neural network model has been developed in [13], where the process is controlled via a neural predictive controller in that case the controller doesn't refer to a mathematical model for the process but consider the black box identification. In our case we are based on the mathematical model for the synthesis of the controller, the obtained results are satisfactory regarding the result obtained in [13]. Another work in the same spirit developed by A. Nikfetrat [14], where the study considers a predictive controller applied to a biological feed-batch process without taking into account the constraints on the input variables and shows an important error in tracking reference trajectories, where the present work gave better results.

\section{Conclusion}

In this paper, the modeling and the control of the biomass growth process are treated. The objective is to control and compare two controllers. The nonlinear model of this process obtained from the mass-balance is transformed to Takagi-Sugeno fuzzy model, which represents exactly the original nonlinear model. Then, a T-S observer is designed to reconstruct the unmeasurable state when the premise variables are not measurable. To ensure the trajectory tracking two controllers are tested. The PDC and the linear quadratic control. The obtained results show that the two controllers are both effective. Finds that the second controller is more stable. In addition, the inputs respect the physical constraints of the process.

In this study, we only focus on the bioprocess control in faulty free case, that is why one interesting future work is to build a fault-tolerant control for this process.

\section{Appendix}

The proof of the Theorem 3 is presented here, we consider the following state estimation error:

$$
e(t)=x(t)-\hat{x}(t)
$$

their dynamic becomes:

$$
\dot{e}(t)=\dot{x}(t)-\dot{\hat{x}}(t)
$$

we take:

$$
\Delta(x, \hat{x}, u)=\left(\sum_{i=1}^{r} h_{i}(z)-\sum_{i=1}^{r} h_{i}(\hat{z})\right)\left(A_{i} x+B_{i} u(t)\right)
$$

the error dynamic then becomes:

$$
\dot{e}=\sum_{i=1}^{r} h_{i}(\hat{z})\left(A_{i}-L_{i} C\right) e(t)+\Delta(x, \hat{x}, u)
$$

if we assume that the term $\Delta(x, \hat{x}, u)$ satisfies the condition of Lipschitz as follows:

$$
\|\Delta(x, \hat{x}, u)\| \leq \alpha\|x-\hat{x}\|
$$

to ensure the convergence of 36, one can consider the following candidate quadratic Lyapunov function:

$$
V(e(t))=e(t)^{T} P e(t)
$$

leads to:

$$
\begin{aligned}
& \dot{V}(e(t))=\sum_{i=1}^{r} h_{i}(\hat{z}) e(t)^{T}\left(\left(A_{i}-L_{i} C\right)^{T} P+P\left(A_{i}-L_{i} C\right)\right)+ \\
& \Delta(x, \hat{x}, u)^{T} P e(t)+e(t)^{T} P \Delta(x, \hat{x}, u)
\end{aligned}
$$

Lemma 1 For two real matrices $X$ and $Y$ of appropriate dimensions, the following inequality is verified:

$$
X^{T} Y+X Y^{T}<X^{T} \Omega^{-1} X+Y \Omega Y^{T}, \Omega>0
$$

Applying the previews lemma to the term:

$\Delta(x, \hat{x}, u)^{T} P e(t)+e(t)^{T} P \Delta(x, \hat{x}, u)$, the derivative of (39) is expressed as:

$\dot{V}(e(t)) \leq e^{T}\left(A_{i}^{T} P-C^{T} L_{i}^{T} P+P A_{i}-P L_{i} C+\alpha^{2} \mathbb{I}+P P\right) e$

If there exists a symmetric and positive definite matrix $Q=Q^{T}$ such that:

$$
A_{i}^{T} P-C^{T} L_{i}^{T} P+P A_{i}-P L_{i} C \leq-Q
$$


leads to:

$$
-Q+\alpha^{2} \mathbb{I}+P P<0
$$

Finally, we apply the Schur complement to 42 we obtain:

$$
\left[\begin{array}{cc}
Q-\alpha^{2} \mathbb{I} & P \\
P & \mathbb{I}
\end{array}\right]>0
$$

Then the proof is completed.

\section{References}

[1] V. Vastemans, M. Rooman, P. Bogaerts, "A robust method for the joint estimation of yield coeffcients and kinetic parameters in bioprocess models", Biotechnology Progress 25 (3), 2009, 606-618. doi:10.1002/btpr.89.

[2] A. Karama, O. Bernard, J.-L. Gouzé," Constrained Hybrid Neural Mod- elling of Biotechnological Processes", International Journal of Chemical Re- 70 actor Engineering 8 (1), 2010, 1-15. doi:https://doi.org/10.2202/ 1542-6580.2117.

[3] V. Grisales, A. Gauthier, G. Roux, "Fuzzy Optimal Control Design for Dis- crete Affine Takagi-Sugeno Fuzzy Models: Application to a Biotechnologi- cal Process", 2006 IEEE International Conference on Fuzzy Systems, 2006, 2369-2376, doi:10.1109/FUZZY.2006.1682030.

[4] E. Herrera, B. Castillo, J. Ramrez, E. C. Ferreira, "Takagisugeno multiple- model controller for a continuous baking yeast fermentation process", ICINCO 2007 - 4th International Conference on Informatics in Control, Automation and Robotics, Proceedings ICSO, 2007, 436-439. doi:10.5220/0001622704360439.

[5] Min-Sen Chiu, Shan Cui, Qing-Guo Wang. “ Internal Model Control Design for Transition Control”, AIChE journal, vol 46, 2000

[6] E. J. Herrera-Liopez, B. Castillo-Toledo, R. Femat, "Fuzzy servo controller 65 for CSTB with substrate inhibition kinetics", Journal of Process Control 22 (6) (2012) 959-967. doi:10.1016/j.jprocont.2012.05.003.

[7] A. M. Nagy Kiss, B. Marx, G. Mourot, G. Schutz, J. Ragot, "Observers de- sign for uncertain Takagi-Sugeno systems with unmeasurable premise vari- ables and unknown inputs. Application to a wastewater treatment plant", 30 Journal of Process Control 21 (7), 2011, 1105-1114. doi:10.1016/j. jprocont.2011.05.001.

[8] M. Bouharkat, M. Ramdani," Fuzzy observer based predictive control of an activated sludge depollution bioprocess", 2013 International Conference on Control, Decision and Information Technologies, CoDIT, 2013, 236-241. doi:10.1109/CoDIT.2013.6689550.

[9] E. Herrera, B. Castilloa, J. Ramrezb, E. C. Ferreirac, "Exact fuzzy observer for a baker's yeast fermentation process", IFAC Proceedings Volumes (IFAC- PapersOnline) 10 (1), 2007, 313318. doi:10.1109/FUZZY.2007.4295502.

[10] S. Aouaouda, M. Chadli, M. T. Khadir, T. Bouarar, "Robust fault tolerant tracking controller design for unknown inputs T-S models with unmeasurable premise variables", Journal of Process Control 22 (5), 2012, 861-872. doi:10.1016/j.jprocont.2012.02.016.
[11] S. Bououden, M. Chadli, H. R. Karimi, "Control of uncertain highly non- linear biological process based on TakagiSugeno fuzzy models", Signal Processing 108 (2015) 195-205. doi:10.1016/j.sigpro.2014.09.011.

[12] S. Carlos-Hernandez, E. N. Sanchez, J. F. Béteau, "Fuzzy observers for anaerobic WWTP: Development and implementation", Control Engineering Practice 17 (6), 2009, 690-702. doi:10.1016/j.conengprac.2008.11. 008.

[13] A. Karama, "Contribution à la modélisation et la commande par réseaux de neurones des bioprocédès", thesis, University Cadi Ayyad, Faculty of Science Semlalia Marrakech, 2004.

[14] A. Nikfetrat, A.R. Vali, V. Babaeipour. "Neural Network Modeling and Nonlinear Predictive Control of a Biotechnological Fed-batch Process", IEEE International Conference on Control and Automation Christchurch, New Zealand, December 9-11, 2009.

[15] Mohd N, Aziz N. "Performance and Robustness Evaluation of Nonlinear Autoregressive with Exogenous Input Model Predictive Control in Controlling Industrial Fermentation Process", Journal of Cleaner Production, 2016.

[16] O. Gehan, E. Pigeon, M. Pouliquen, L. Fall, R. Mosrati, "Nonlinear control of dissolved oxygen level for Pseudomonas putida bacterium fermentation", 152016 IEEE Conference on Control Applications, CCA 2016 (2016) 1215- 1220 doi:10.1109/CCA.2016.7587972.

[17] K. Tanaka, H. O. Wang, "Fuzzy Control Systems Design and Analysis", John Wiley and Sons, Inc., New York, USA, 2001. doi:10.1002/0471224596.

[18] Saifia, D., Chadli, M., Labiod, S., and Guerra, T. M., " Robust $H \infty$ Static Output Feedback Stabilization of T-S Fuzzy Systems Subject to Actuator Saturation", Int. J. Control Autom. Syst. $10(3), 613-622,2012$

[19] Hassan.K.Khali. "Nonlinear Systems", second edition, Prentice Hall. United States of America, 1996.

[20] Guechi, El-Hadi, Lauber, Jimmy, Dambrine, Michel, et al., "PDC control design for non-holonomic wheeled mobile robots with delayed outputs", J. Intell. Robot. Syst. 60(3-4), 395-414, 2010

[21] Xu, Bin, Wang, Shixing, Gao, Daoxiang, et al., "Command filter based robust nonlinear control of hypersonic aircraft with magnitude constraints on states and actuators", J. Intell. Robot. Syst. 73(1-4), 233-247, 2014

[22] Zhu, Senqiang et Wang, Danwei., " Adversarial ground target tracking using UAVs with input constraints”, J. Intell. Robot. Syst. 65(1-4), 521-532, 2012

[23] M. Abyad, A. Karama, A. Khalloq, "Fuzzy Takagi-Sugeno Based Modelling and Control For an Alcoholic Fermentation Process", 3rd International Conference on Electrical and Information Technologies ICEIT, Rabat, Morocco, 2017.

[24] P. Bergsten, R. Palm, D. Driankov, "Fuzzy observers", 10th IEEE International Conference on Fuzzy Systems. (Cat No.01CH37297) 2, 2001, doi:10.1109/FUZZ.2001.1009051.

[25] Bastin G. and Dochain D. "On-line estimation and adaptive control of bioreactors". Elsevier, Amsterdam, 1990. 